\title{
Editorial
}

Jean-François Hardy MD FRCPC, Sylvain Belisle MD FRCPC, Normand Tremblay MD FRCPC

\section{Cardiac anaesthesia: a perspective for the 1990's}

But is it really? The arguments in favour of or against early extubation in patients following coronary artery surgery have been reviewed recently. ${ }^{5,6}$ Most, but not all, ${ }^{7}$ would accept that early extubation does not apply to patients coming for valvular surgery, and agree on the identification of the candidate for early extubation outlined by Higgins, based on preoperative, intraoperative and postoperative characteristics known to predict postoperative morbidity (especially respiratory complications) and mortality. ${ }^{5}$ Essentially, the candidate is younger than $70 \mathrm{yr}$, has adequate ventricular function, is free of severe mitral, aortic or systemic disease, has an uncomplicated intraoperative course, and presents a normal cardiovascular, neurological, renal and coagulation status in the immediate postoperative period. Unfortunately, in this Institution ${ }^{8}$ as in many other tertiary care centres, "healthy" CABG patients are in ever-decreasing numbers. Even so, Hall rightly reminds us that routine is no excuse not to offer these patients the alternative of early extubation.

Drugs such as midazolam, propofol, alfentanil and sufentanil, with the concomitant administration of volatile anaesthetic agents and/or measures to control the sympathetic nervous system, can be tailored to provide an anaesthetic allowing early extubation of the trachea. However, control of the stress response after surgery, in view of reducing the incidence of perioperative ischaemia, is essential. ${ }^{9}$ Hall suggests that the pharmacokinetic profile of propofol and sufentanil by infusion allows the provision of close to ideal sedation/analgesia in this setting. One may argue that other drugs such as midazolam, alfentanil, or non-narcotic analgesics might prove equally useful. Intraoperative and postoperative analgesia with a thoracic epidural infusion of bupivacaine and sufentanil has been shown to improve recovery time, as well as pulmonary and cardiac outcome after CABG when compared with intravenous postoperative pain treatment after intraoperative general anaesthesia with midazolam and sufentanil. ${ }^{10}$ Therefore, the best regimen to achieve control of postoperative stress and ischaemia remains to be determined. Clinicians must keep in mind that optimal benefits on outcome will not be achieved unless the postopera-
From the Department of Anesthesiology, Montreal Heart Institute, 5000 Belanger Street East, Montreal, Quebec. 
tive pain relief afforded to patients is utilized to enhance early mobilization and promote nutritional intake.* Thus, early extubation is but a part of the global patient management strategy that relies not only on anaesthetic technique, but also on expeditious surgery, proper administrative management of the transition from the immediate recovery area to the intermediate care unit, and appropriate postoperative care.

Proponents of early extubation usually invoke the high costs of hospital beds in the Intensive Care Unit (ICU) and the expected savings derived from a shortened stay in the ICU. This type of economic evaluation of health care activities has been termed the cost minimization approach. " Cost minimization assumes identical outcomes for the approaches under comparison, a rare occurrence in medicine, and is therefore of limited usefulness. The limitations of two other economic evaluations, costeffectiveness and cost-benefit approaches, have led to the development of a fourth type of economic evaluation, cost-utility analysis. Cost-utility analysis attempts to identify and compare the costs of competing treatment routes producing a given measure of health gain (e.g., qualityadjusted life year; QALY). ${ }^{11}$ While some tentative estimates of the cost of CABG per QALY are available, the effect of early extubation on this cost remains to be calculated, but is probably minimal.

A recent multicentre study in the Canadian health care system identified considerable differences among four hospitals in the postoperative utilization of critical care services by cardiac surgery. ${ }^{12}$ Gross outcome (measured by mortality and hospital lengths of stay) was similar for similar patient "input," despite lengths of stay in the ICU that varied from 2.3 to 8.7 days. The authors concluded that, in order to improve resource use in this group of patients, assessments of utilization must focus on more detailed specific issue than unit length of stay, and must include factors such as the availability of intermediate care areas ("step-down" unit), the management system of the unit, the chronic health status of patients coming to surgery, and the operative procedures performed. For example, in the absence of intermediate care facilities, requirements for monitoring alone may delay discharge from the ICU after cardiac surgery, ${ }^{12}$ while, at the same time, meaningful conclusions about the effectiveness and safety of pulmonary artery catheterization are lacking. ${ }^{13}$ Since the cost of critical care is related directly to both the duration of stay and the interventions utilized, ${ }^{14}$ physicians must attempt to provide some evidence for the

* Kehlet $H$. Postoperative pain relief - a look from the other side. Presented at the Eighteenth Annual Meeting of the American Society of Regional Anesthesia. Seattle, Washington, May $15,1993$. efficacy of these practices. Thus, early extubation is only one of the numerous factors in the complex equation relating cost to the outcome of health care activities.

Several other practices in cardiac anaesthesia will require close scrutiny in the years to come. These include, for example, the methods employed to preserve the brain, the myocardium, the kidneys and the physiological cascades (including coagulation) activated by cardiopulmonary bypass (CPB). Individuals with the skills of epidemiology, logistics, economics and ethics will be essential to provide data on outcome, delivery and cost of therapy provided by anaesthetists. ${ }^{15}$ Much more work is needed in the field of cerebral protection if transient and permanent neurological deficits, probably the most damaging effects of CPB, are to be avoided. Protection of the myocardium during surgery is the subject of considerable debate among our surgical colleagues. Recent experience with warm blood cardioplegia and normothermic CPB shows that myocardial preservation techniques must be evaluated not only in terms of their primary effectiveness on cardiac muscle, but also on their systemic effects, beneficial or deleterious. In the case of normothermic CPB, the incidence of permanent neurological deficits is of particular interest. ${ }^{16}$ In addition, normothermia during CPB will act upon platelet function, renal function, and produce ill-defined effects on physiological cascades. The effectiveness of sophisticated monitoring to prevent myocardial or cerebral ischaemia, such as transoesophageal echocardiography, continuous pulmonary artery oximetry, computerized multilead electroencephalography and continuous blood gas analysis during CPB will have to be demonstrated. Similarly, the routine use of membrane oxygenators, arterial line filters, leukocyte depletion filters, new materials for CPB circuits, the effectiveness of pulsatile CPB, or the need for CPB itself must be examined. Clinically helpful intraoperative monitors of coagulation may be developed in the next few years, that will allow for the optimal use of drugs such as antifibrinolytics to decrease excessive postoperative bleeding and transfusion of homologous blood products in our patients. So, where is cardiac anaesthesia going in the 1990's? The perennial driving force of progress in medicine is the reduction of mortality and morbidity. Advances in anaesthesia, such as high-dose opioid techniques, have permitted successful operations on much sicker patients and contributed to the development of cardiac surgery. As a consequence, mortality has been transferred from the intraoperative to the postoperative period. Having mastered anaesthetic techniques and haemodynamic control, cardiac anaesthetists must now move towards more global aspects of care to pursue the reduction of mortality and morbidity achieved intraoperatively. Progress in this direction requires that new information 
on drugs, equipment and techniques be integrated thoughtfully in the body of existing safe practices. As reviewed by Hall, sufficient drugs and pharmacological knowledge are available to provide safe anaesthesia and rapid tracheal extubation after surgery in the subset of "healthy" patients coming for CABG. Only if included in a global and preconcerted approach to patient care do we believe rapid extubation in this group of patients is normally beneficial, and thus desirable. We should not forget, however, lest we relapse in a state of "mindlessness," that the timing of extubation is only one of the numerous aspects of patient care we must reassess continuously.

\section{Que réservent les années 1990 à l'anesthésie cardiaque?}

Dans son livre " Mindfulness ", Ellen J. Langer réfléchit sur cette habitude que nous avons de faire confiance à des méthodes systématiques non éprouvées. Cette coutume empêche toute réflexion et nous mène à un état d'insouciance caractériseée qui constitue un terrain fort aux accidents. ' La mise en cause constante du bien-fondé de nos tâches quotidiennes nous occupe l'esprit et nous maintient en état d'alerte. En anesthesie cardiaque, une de ces habitudes bien ancrees consiste à administrer de fortes doses d'opiacés et de prolonger la ventilation en postopératoire. Nous devons feliciter Hall pour sa remise en cause de l'extubation tardive apres pontage aortocoronariens (PAC) sous circulation extracorporelle (CEC). ${ }^{2}$ Dans le Journal, Hall refute aujourd'hui l'argument selon lequel les effets secondaires des drogues utilisées par l'anesthésiste pendant l'operation doivent lui dicter sa conduite postopératoire. Bien appuyé sur la pharmacocinétique, il décrit des techniques de rechange qui permettent l'extubation précoce si elle présente des avantages pour le patient.

En 1969, Lowenstein rapportait que de fortes doses de morphine $i v$ prévenaient la dépression cardiaque défavorable induite par les anesthésiques en usage, plus particulièrement le thiopentone et l'halothane, chez les patients soumis à une chirurgie a coeur ouvert de valvulopathies acquises et plus specialement celles de la valve aortique. ${ }^{3}$ Avant 1966 , plusieurs ecoles preconisaient la ventilation mécanique postoperatoire systema- tique pour les opérés sous CEC, de façon à favoriser l'administration de fortes doses de morphine. Cette pratique non seulement permettait de mener à bien l'opération elle-même, mais aussi de passer plus facilement à la ventilation mécanique postopératoire. Toutefois, déjà en 1971, Lowenstein reconnaissait que l'arrivée en masse de la chirurgie des coronaires nécessiterait une réévaluation de cette pratique parce que les futurs patients ne souffraient pas nécessairement d'une baisse chronique du débit cardiaque. ${ }^{4} \mathrm{La}$ controverse extubation précoce vs extubation tardive existe donc depuis les débuts de la chirurgie cardiaque.

Mais est-ce encore vrai? On a récemment révisé le pour et le contre de l'extubation précoce après le PAC. ${ }^{5,6}$ Tous les auteurs, à quelques exceptions près, ${ }^{7} \mathrm{~s}$ 'accordent pour dire que l'extubation précoce ne convient pas à la chirurgie valvulaire. Ils reconnaissent la validité des critères de Higgins pour lidentification des candidats à l'extubation précoce. Ces critères de morbidité (surtout les complications respiratoires) et de mortalité sont basés sur les caractéristiques préopératoires, peropératoires et postopératoires du patient. ${ }^{5}$ En résumé, le candidat a moins de 70 ans; sa fonction ventriculaire est adéquate; il est exempt de maladies systémiques ou de valvulopathie aortique ou mitrale grave; il a subi l'intervention sans complications; à la période postopératoire immédiate, sa condition cardiovasculaire, neurologique, rénale est stable; il coagule bien. Malheureusement, chez nous, ${ }^{8}$ comme dans plusieurs centres de soins tertiaires, les candidats non tarés au PAC se font de plus en plus rares. Encore là, Hall insiste à juste titre pour nous rappeler que l'habitude est une bien mauvaise excuse pour refuser à ces patients les bienfaits de l'extubation précoce.

Des drogues telles que le midazolam, le propofol, l'alfentanil et le sufentanil, associées à des agents volatils et à des bloqueurs du système sympathique peuvent être titrées de façon à produire une bonne anesthésie suivie d'une extubation rapide. Cependant, afin de diminuer l'incidence de l'ischémie périopératoire, il est essentiel de contrôler la réponse au stress. ${ }^{9}$ Hall suggère que le profil pharmacocinétique du propofol et du sufentanil en perfusion offrent dans ces conditions la meilleure analgésie et la meilleure sédation possibles. On peut toujours répondre que d'autres drogues comme le midazolam, l'alfentanil ou les analgésiques non opiacés peuvent aussi faire l'affaire. Il a été aussi démontré que l'analgésie peropératoire et postopératoire réalisée par une perfusion péridurale de bupivacaine et de sufentanil diminuait le temps de récupération et améliorait le pronostic pulmonaire et cardiaque après le PAC, comparativement à une perfusion postopératoire intraveineuse de sufentanil qui suivait une anesthésie au midazolam-sufentanil. ${ }^{10}$ On ne peut présentement identifier avec certitude la meilleure 
méthode de contrôle du stress et de lischémie postopératoires. Le clinicien doit toujours avoir à l'esprit que les meilleurs résultats ne sont possibles que si l'efficacité de l'analgésie postopératoire est au service de la mobilisation précoce et l'apport nutritionnel.* L'extubation précoce n'est donc qu'une partie de la stratégie de la prise en charge globale. Cette stratégie ne s'apuie pas seulement sur une bonne technique anesthésique, mais aussi sur une chirurgie expéditive, une gestion appropriée de la période de transition qui sépare l'aire de récupération immédiate de celle de soins postopératoires compétents. ${ }^{7}$

Les adeptes de l'extubation précoce invoquent généralement l'argument des coûts élevés du séjour hospitalier en unité de soins intensifs (USI) et les économies réalisées lorsqu'on diminue sa durée. On a appelé ce type d'évaluation économique de l'activité sanitaire " approche de minimisation des coûtes " (cost minimization approach)." La minimisation des coûts assume que les résultats sont identiques pour les méthodes qu'on compare, ce qui survient rarement en médecine, et limite ainsi son utilisation. Les limites inhérentes à deux autres formules d'évaluation économique, coût-efficacité et coûtbénéfice on mené à une quatrième formule d'évaluation à caractère économique, l'analyse coût-utilité. Cette dernière tente d'identifier et de comparer les coûtes de deux méthodes therapeutiques destinées à procurer un bénéfice pour la santé en terme de qualité de vie ajustée au nombre d'années. " Lorsqu'on évalue le coût du PAC sous ce rapport, la part de l'extubation précoce n'a pas été mesurée spécifiquement mais pourrait être négligeable.

Une étude multicentrique menée à lintérieur du système de soins de santé canadien a identifié dans quatre hôpitaux des différences importantes quant à l'utilisation des soins intensifs après la chirurgie cardiaque. ${ }^{12} \mathrm{Le}$ résultat brut (la mesure de la mortalité et de la longueur du séjour hospitalier) était le même pour une série de patients identiques, malgré des durées de séjour à l'USI variant de 2,3 à 8,7 jours. Les auteurs concluent que, pour ce groupe de patients, l'évaluation de l'utilisation doit se concentrer sur d'autres critères que celui de la durée du séjour à l'USI. Cette évaluation doit inclure la disponibilité d'unités de soins intermédiaires, le système de gestion de l'unité, l'état de santé de l'opéré, et les interventions effectuées. Par exemple, en absence d'une unité de soins intermédiaires, les besoins du monitorage seuls peuvent, après la chirurgie, retarder le départ de l'USI, alors qu'en même temps on est incapable de tirer des conclusions valable sur l'efficacité et la sécurité d'un cathéter de Swann-Ganz. ${ }^{15}$ Comme le coût des soins intensifs

* Kehlet $H$. Postoperative pain relief - a look from the other side. Présenté à la $18 \mathrm{e}$ réunion annuelle de l'American Society of regional Anesthesia, Seattle, Washington, le 15 mai 1993. dépend autant de la durée du séjour que du type dintervention pratiquée, ${ }^{14}$ il appartient aux médecins de prouver que ces pratiques sont efficaces. Ainsi, l'extubation précoce n'est qu'un des nombreuses facteurs de l'équation complexe qui relie le coût aux résultats des interventions thérapeutiques.

A l'avenir, plusieurs autres pratiques de l'anesthésie cardiaque nécessiteront un examen approfondi. Il faut mentionner, par exemple, les méthodes de protection du cerveau, du myocarde, du rein et la cascade physiologique activée par la CEC, incluant la coagulation. Les épidémiologistes, logisticiens, économistes et éthiciens devront nous fournier des données sur les résultats, l'administration et le coût des thérapies dispensées par les anesthésistes. ${ }^{15}$ Beaucoup plus de travail doit être effectuée dans le domaine de la protection cérébrale, si on veut éviter les déficits neurologiques transitoires et permanents qui constituent les effets les plus nocifs de la CEC. Chez nos collègues chirurgiens, la protection du myocarde pendant la chirurgie demeure un sujet fort controversé. Les expériences récentes de la cardioplégie chaude et de la CEC normothermique montrent que les techniques de préservation myocardique doivent être évaluées non seulement en rapport avec leur efficacité sur le coeur, mais aussi en rapport avec leurs effets systémiques, qu'il soient favorables ou nuisibles. En ce qui concerne la CEC normothermique, son influence sur l'incidence des dommages neurologiques permanents est particulièrement intéressante. ${ }^{16}$ De plus, pendant la CEC, la normothermie influera sur la fonction plaquettaire, et rénale tout en ayant des effets mal définis sur les cascades physiologiques. On devra démontrer l'efficacité du monitorage de pointe destiné à prévenir, pendant la CEC, l'ischémie myocardique et cérébrale comme l'échocardiographie transoesophagienne, l'oxymétrie artérielle pulmonaire continue, l'électroencéphalographie informatisée à canaux multiples et l'analyse continue des gaz artériels. De la même façon, l'utilisation systématique de l'oxygénateur à membrane, de filtres de canalisations artérielles et de déplétion leucocytaire, les nouveaux matériaux des circuits de CEC, l'efficacité de la CEC pulsatile, et l'utilisation même de la CEC devront être revus. Des moniteurs de coagulation peropératoires pourraient voir le jour dans les années qui viennent; ils permettront l'utilisation optimale, entre autres, des antifibrinolytiques pour diminuer le saignement postopératoire et les transfusions de produits sanguins homologues.

Quelle direction prendra l'anesthésie cardiaque dans les années 1990? En médecine, la diminution de la morbidité et de la mortalité représente l'énergie motivatrice du progrès. En anesthésie, le développement de techniques, comme l'administration d'opiacés à hautes doses, a permis de réussir des interventions chez des sujets beaucoup 
plus malades et a ainsi contribué aux progrès de l'anesthésie. Il en est résulté qu'une partie de la mortalité peropératoire s'est transposée à la période postopératoire. Après avoir maitrisé les techniques de l'anesthésie et contrôlé l'hémodynamie, les anesthésistes cardiaques doivent s'intéresser à la totalité des soins s'ils veulent poursuivre leur objectif. Des progrès ne seront réalisés qu'avec l'intégration aux modalités d'exercice actuelles des nouvelles connaissances pharmacologiques, technologiques et pratiques. Comme le fait remarquer Hall, nos connaissances des nouvelles drogues et de leur pharmacologie suffisent pour procurer une anesthésie sécuritaire et une extubation précoce chez une catégorie de patients non tarés subissant un pontage aortocoronarien. Ce n'est qu'avec une approche globale et arrêtée à l'avance que l'extubation précoce, à notre avis, profitera à cette classe de patients. Nous ne devons pas oublier, toutefois, au risque de retomber dans l'insouciance, que le moment de l'intubation n'est qu'un seul des aspects des soins qu'il faudra réévaluer constamment.

\section{References}

1 Langer EJ. Mindfulness. Menlo, California: Addison Wesley, 1990.

2 Hall RI. Anaesthesia for coronary artery surgery - a plea for a goal-directed approach. Can J Anaesth 1993;

40: 1178-94.

3 Lowenstein E, Hallowell P, Levine FH, Daggett WM, Austen WG, Laver $M B$. Cardiovascular response to large doses of intravenous morphine in man. $\mathbf{N}$ Engl $\mathbf{J}$ Med 1969; 281: 1389-93.

4 Lowenstein E. Morphine "anesthesia" - a perspective (Editorial). Anesthesiology 1971; 35: 563-5.

5 Higgins TL. Pro: Early endotracheal extubation is preferable to late extubation in patients following coronary artery surgery. J Cardiothorac Vasc Anesth 1992; 6: 488-93.

6 Siliciano $D$. Con: Early extubation is not preferable to late extubation in patients undergoing coronary artery surgery. J Cardiothorac Vasc Anesth 1992; 6: 494-8.

7 Chong $J L$, Pillai R, Fisher A, Grebenik C, Sinclair $M$, Westaby $S$. Cardiac surgery: moving away from intensive care. Br Heart J 1992; 68: 430-3.

8 Tremblay NA, Hardy JF, Perrault J, Carrier M. A simple classification of the risk in cardiac surgery: the first decade. Can J Anaesth 1993; 40: 103-11.

9 Mangano DT, Siliciano D. Hollenberg $M$, et al. Postoperative myocardial ischemia. Therapeutic trials using intensive analgesia following surgery. Anesthesiology 1992; 76: $342-53$.

10 Liem TH, Hasenbos MAWM, Booij LHDJ, Gielen MJM. Coronary artery bypass grafting using two different anesthetic techniques: Part 2. Postoperative outcome. J Cardiothorac Vasc Anesth 1992; 6: 156-61.
11 Maynard A. Economic evaluation and cardiac surgery. In: Yacoub M, Pepper JR (Eds.). Annual of Cardiac Surgery, Philadelphia: Current Science Ltd. 1992.

12 Mazer CD, Byrick RJ, Sibbald WJ, et al. Postoperative utilization of critical care services by cardiac surgery: a multicenter study in the Canadian healthcare system. Crit Care Med 1993; 21: 851-9.

13 A report by the American Society of Anesthesiologists Task Force on Pulmonary Artery Catheterization. Practice guidelines for pulmonary artery catheterization. Anesthesiology 1993; 78: 380-94.

14 Taylor GJ, Mikell FL, Moses HW, et al. Determinants of hospital charges for coronary artery bypass surgery: the economic consequences of postoperative complications. Am J Candiol 1990; 65: 309-13.

15 Lanier $W L$, Warner MA. New frontiers in anesthesia research. Assessing the impact of practice patterns on outcome, health care delivery, and cost. Anesthesiology 1993; 78: 1001-4.

16 Guyton RA. Warm blood cardioplegia: benefits and risks. Ann Thorac Surg 1993; 55: 1071-2. 